\title{
Epigenetic Modifications in Placenta are Associated with the Child's Sensitization to Allergens
}

\author{
Hani Harb, ${ }^{1,2,3}$ Bilal Alashkar Alhamwe ${ }^{10},{ }^{1,2,4}$ Nathalie Acevedo, ${ }^{5,6}$ Paolo Frumento, ${ }^{7}$ \\ Catharina Johansson, ${ }^{5}$ Lisa Eick, ${ }^{1}$ Nikos Papadogiannakis, ${ }^{8}$ Johan Alm, ${ }^{5}$ Harald Renz $@$, ${ }^{1,2}$ \\ Daniel P. Potaczek $(\mathbb{1}),{ }^{1,2,9}$ and Annika Scheynius ${ }^{5,10}$ \\ ${ }^{1}$ Institute of Laboratory Medicine, Member of the German Center for Lung Research (DZL) and the Universities of Giessen and \\ Marburg Lung Center (UGMLC), Philipps-University Marburg, Germany \\ ${ }^{2}$ International Inflammation (in-VIVO) Network, Worldwide Universities Network (WUN), West New York, NJ, USA \\ ${ }^{3}$ Division of Immunology, Boston Children's Hospital, Harvard Medical School, Boston, MA, USA \\ ${ }^{4}$ College of Pharmacy, International University for Science and Technology (IUST), Daraa, Syria \\ ${ }^{5}$ Department of Clinical Science and Education, Karolinska Institutet, and Sachs' Children and Youth Hospital, Södersjukhuset, \\ Stockholm, Sweden \\ ${ }^{6}$ Institute for Immunological Research, University of Cartagena, Cartagena, Colombia \\ ${ }^{7}$ Unit of Biostatistics, Institute of Environmental Medicine, Karolinska Institutet, Stockholm, Sweden \\ ${ }^{8}$ Department of Laboratory Medicine, Division of Pathology, Section of Perinatal Pathology, Karolinska Institutet and \\ Karolinska University Hospital Huddinge, Stockholm, Sweden \\ ${ }^{9}$ John Paul II Hospital, Krakow, Poland \\ ${ }^{10}$ Clinical Genomics, Science for Life Laboratory, Stockholm, Sweden
}

Correspondence should be addressed to Daniel P. Potaczek; daniel.potaczek@staff.uni-marburg.de

Hani Harb and Bilal Alashkar Alhamwe contributed equally to this work. Daniel P. Potaczek and Annika Scheynius also contributed equally to this work.

Received 30 December 2018; Revised 19 February 2019; Accepted 12 March 2019; Published 17 April 2019

Academic Editor: Oscar Palomares

Copyright (c) 2019 Hani Harb et al. This is an open access article distributed under the Creative Commons Attribution License, which permits unrestricted use, distribution, and reproduction in any medium, provided the original work is properly cited.

Prenatal environmental exposures are considered to contribute to the development of allergic sensitization by epigenetic mechanisms. The role of histone acetylation in the placenta has not been examined yet. We hypothesized that placental histone acetylation at the promoter regions of allergy-related immune regulatory genes is associated with the development of sensitization to allergens in the child. Histones $\mathrm{H} 3$ and $\mathrm{H} 4$ acetylation at the promoter regions of 6 selected allergy-related immune regulatory genes was assessed by a chromatin immunoprecipitation assay in 173 term placentas collected in the prospective birth-cohort ALADDIN. The development of IgE sensitization to allergens in the children was followed from 6 months up to 5 years of age. We discovered significant associations of histone acetylation levels with decreased risk of allergic sensitization in 3 genes. Decreased risk of sensitization to food allergens was associated with higher $\mathrm{H} 3$ acetylation levels in placentas at the IFNG and SH2B3 genes, and for $\mathrm{H} 4$ acetylation in HDAC4. Higher HDAC4 $\mathrm{H} 4$ acetylation levels were also associated with a decreased risk of sensitization to aeroallergens. In conclusion, our results suggest that acetylation of histones in placenta has a potential to predict the development of sensitization to allergens in children.

\section{Introduction}

During the last decades the prevalence of allergic sensitization and allergic diseases has increased worldwide, particularly in children [1,2]. One hypothesis to explain this allergy epidemic is that environmental and lifestyle conditions modify the epigenome of immune cells, so the immune response is skewed to proallergic profiles [3-5]. 
Prenatal or early childhood exposures to environmental factors, such as living in urban or rural/farming areas can affect the programming of the immune system and are thus risk factors for development of subsequent allergic diseases [6]. Epigenetic modifications have been postulated as an important mechanism mediating these effects [7-10], which has been demonstrated mostly by human studies investigating DNA methylation [11, 12]. Previously, it has been found that DNA methylation levels within the CD14 promoter region are lower in placentas of mothers living on a farm, suggesting that epigenetic regulation of CD14 early in life might be involved in the protective effect of "living on a farm", with regard to allergy development [13].

Much less in this context is known on the eventual role of epigenetic modifications of histones in allergy development. Unlike DNA methylation, histone modifications, for example, histone acetylation, methylation, or phosphorylation, are biochemical changes affecting not the nucleic acid itself but lysine residues on histones. Increased acetylation of histones $\mathrm{H} 3$ and $\mathrm{H} 4$ is typically associated with better accessibility of promoters to transcriptional machinery and thereby higher gene expression [5]. Previous studies have shown that changes in histone acetylation levels can affect polarization of T helper type 2 (Th2) cells/response [14].

In the present study, we hypothesized that acetylation of $\mathrm{H} 3$ or $\mathrm{H} 4$ histones in the promoter regions of potentially allergy-related immune regulatory genes in placenta tissue is associated with the development of sensitization to food and airborne allergens in the child early in life. We nested our study within the prospective birth-cohort ALADDIN (Assessment of Lifestyle and Allergic Disease During INfancy), which consists of families with different lifestyles, anthroposophic, partly anthroposophic or conventional [15]. Anthroposophic lifestyle is mainly characterized by organic diet with live lactobacilli, restrictive use of antibiotics and vaccine, and home delivery $[15,16]$. The ALADDIN cohort was designed to elucidate why children in anthroposophic families are less sensitized to allergens compared to those living in families with a conventional lifestyle [15, 16]. The results might indicate that acetylation of histones in allergy-related immune regulatory genes in placenta has a potential to predict the development of sensitization to allergens in children early in life.

\section{Materials and Methods}

2.1. Study Population: The ALADDIN Cohort. Mothers and their children in this study are part of the prospective birthcohort ALADDIN, which consists of families with different lifestyles $[15,16]$. A total of 330 families were recruited at anthroposophic and conventional healthcare centers in the Stockholm area between September 2004 and November 2007. Families were enrolled in the study at gestational weeks 25-37 (median 30). The lifestyle groups were classified based on choice of maternal-child health centers and parental responses to a questionnaire two months after the birth of the child as described in more detail elsewhere [15]. Inclusion criteria for the present study were not severe illness before or during pregnancy, $\geq 36$ weeks of gestation, and availability of snap frozen placenta specimens stored at $-80^{\circ} \mathrm{C}$ which had not previously been thawed for use in other studies ending with 173 placentas (Table 1). This study was conducted in accordance with the Declaration of Helsinki and was approved by the Regional Ethical Review Board in Stockholm (project Dnr 2010/1811-32). All parents gave their written informed consent for inclusion before they participated in the study.

2.2. Determination of Allergen Sensitization. Blood samples were obtained from parents at inclusion in the study and from the child at $6,12,24$, and 60 months of age. Samples were collected in heparin tubes and plasma was stored at $20^{\circ} \mathrm{C}$. Parental sensitization was analyzed by ImmunoCAP Phadiatop $^{\mathrm{TM}}$ for IgE to a mix of 11 aeroallergens. Available blood samples from the children at 6,12, and 24 months of age were analyzed by ImmunoCAP tests for IgE to cow's milk, hen's egg, peanut, cat, dog, birch, and timothy. At 60 months, a food mix ( $\mathrm{fx} 5$ ) and Phadiatop ${ }^{\mathrm{TM}}$ were used. If $\mathrm{fx} 5$ was positive, the allergens cow's milk, hen's egg, peanut, codfish, wheat flour, and soybean were separately analyzed and if Phadiatop $^{\text {TM }}$ was positive, cat, dog, horse, birch, timothy, mugwort, Cladosporium, Dermatophagoides farinae, and D. pteronyssinus (all kits from Thermo Fisher Scientific, Uppsala, Sweden). Allergen specific IgE levels $\geq 0.35 \mathrm{kU}_{\mathrm{A}} / \mathrm{L}$ were categorized as IgE sensitization.

\subsection{Collection of Placenta Specimens and Histopathologic} Examination. The placentas $(\mathrm{n}=173)$ were collected by midwifes directly after birth, put on ice, and sent to the Karolinska University Hospital Solna. From each placenta, a crosssectional sample about $0.5 \mathrm{~cm}$ thick, $1.5 \mathrm{~cm}$ wide, and spanning the whole thickness of the placenta was cut near the umbilical cord, quickly washed two times in phosphate buffered saline (PBS) to remove as much blood as possible and then snap frozen on dry ice and stored at $80^{\circ} \mathrm{C}$. These samples were later used for histone acetylation analyses.

The placentas were also subjected to routine histopathological examination. Two placental tissue biopsies, one from the vicinity of the umbilical cord and one from the periphery of the placenta, about $0.5 \mathrm{~cm}$ thick and spanning the whole thickness of the placenta, a piece of the membranes, and a piece of the umbilical cord were obtained. All specimens were washed with PBS, fixed in formalin, paraffin-embedded, and evaluated on routine haematoxylin and eosin-stained sections. In two cases inadequate material had been sampled leaving 171 placentas to be finally analyzed for histopathology. An experienced perinatal pathologist (NP) who was blinded to the demographic data for the participating families (see Table 1) examined all slides. The presence of chorioamnionitis, vasculitis, funisitis, and villitis was recorded. Chorioamnionitis, irrespective of grading, was defined as presence of polymorphonuclear leucocytes in subchorionic plate or in amniochorion. Vasculitis was defined as the presence of leukocytes in the vessel wall of chorionic plate or umbilical vessels. Funisitis was defined as the presence of leukocytes 
TABLE 1: Demographic data for the participating families and sensitization to allergens.

\begin{tabular}{|c|c|c|c|c|c|}
\hline & $\begin{array}{l}\text { Anthroposophic } \\
\qquad \mathrm{N}=25\end{array}$ & $\begin{array}{c}\text { Partly } \\
\text { anthroposophic } \\
\mathrm{N}=105\end{array}$ & $\begin{array}{l}\text { Anthroposophic } \\
\text { and partly } \\
\text { anthroposophic } \\
\mathrm{N}=130\end{array}$ & $\begin{array}{l}\text { Non- } \\
\text { anthroposophic } \\
\mathrm{N}=43\end{array}$ & $\mathrm{p}^{*}$ \\
\hline \multicolumn{6}{|l|}{ Parents } \\
\hline Mother's age (years) & $30(23-32)$ & $31(28-34)$ & $31(27-34)$ & $30(28-33)$ & 0.468 \\
\hline Mother sensitized to aeroallergens ${ }^{\mathrm{a}}$ & $5 / 25(20 \%)$ & $29 / 105(28 \%)$ & $34 / 130(26 \%)$ & $14 / 43(33 \%)$ & 0.537 \\
\hline Father sensitized to aeroallergens ${ }^{\mathrm{a}}$ & $8 / 23(35 \%)$ & $45 / 100(45 \%)$ & $53 / 123(43 \%)$ & $14 / 40(35 \%)$ & 0.473 \\
\hline \multicolumn{6}{|l|}{ Mother during pregnancy } \\
\hline \multicolumn{6}{|l|}{ Parity } \\
\hline First & $7 / 25(28 \%)$ & $46 / 103(45 \%)$ & $53 / 128(41 \%)$ & $17 / 43(40 \%)$ & 0.971 \\
\hline Second & $9 / 25(36 \%)$ & $40 / 103(39 \%)$ & $49 / 128(38 \%)$ & $18 / 43(42 \%)$ & 0.814 \\
\hline Third (fourth or fifth) & $9 / 25(36 \%)$ & $17 / 103(17 \%)$ & $26 / 128(20 \%)$ & $8 / 43(19 \%)$ & 0.982 \\
\hline \multicolumn{6}{|l|}{ Child } \\
\hline Sex (female) & $13 / 25(52 \%)$ & $52 / 105(50 \%)$ & $65 / 130(50 \%)$ & $28 / 43(65 \%)$ & 0.122 \\
\hline Birth weight (g) & $\begin{array}{c}3550 \\
(3355-3760)\end{array}$ & $\begin{array}{c}3585 \\
(3345-3950)\end{array}$ & $\begin{array}{c}3568 \\
(3348-3939)\end{array}$ & $\begin{array}{c}3510 \\
(3312-4010)\end{array}$ & 0.648 \\
\hline Gestational age at birth (completed weeks) & $40(39-41)$ & $40(39-41)$ & $40(39-41)$ & $39(38-40)$ & 0.009 \\
\hline \multicolumn{6}{|l|}{ Child sensitized to } \\
\hline \multicolumn{6}{|l|}{ Food allergens at (Girls n/N; Boys n/N \% p*) } \\
\hline $6 \mathrm{mo}^{\mathrm{b}}(4 / 705.7 ; 6 / 639.5 \mathrm{p}=0.52)$ & $0 / 16(0 \%)$ & $4 / 84(5 \%)$ & $4 / 100(4 \%)$ & $6 / 33(18 \%)$ & 0.022 \\
\hline $12 \mathrm{mo}^{\mathrm{b}}(8 / 6811.8 ; 10 / 5916.9 \mathrm{p}=0.45)$ & $0 / 13(0 \%)$ & 9/77 (12\%) & 9/90 (10\%) & $9 / 37(24 \%)$ & 0.068 \\
\hline $2 \operatorname{yrs}^{\mathrm{b}}(7 / 6810.3 ; 13 / 6221.0 \mathrm{p}=0.14)$ & $2 / 15(13 \%)$ & 9/79 (11\%) & $11 / 94(12 \%)$ & $9 / 36(25 \%)$ & 0.108 \\
\hline $5 \mathrm{yrs}^{\mathrm{c}}(13 / 6021.7 ; 8 / 5215.4 \mathrm{p}=0.47)$ & $1 / 16(6 \%)$ & $10 / 69(14 \%)$ & $11 / 85(13 \%)$ & $10 / 27(37 \%)$ & 0.012 \\
\hline \multicolumn{6}{|l|}{ Aeroallergens at } \\
\hline $6 \mathrm{mo}^{\mathrm{d}}(3 / 644.7 ; 0 / 530.0 \mathrm{p}=0.25)$ & $0 / 13(0 \%)$ & $2 / 72(3 \%)$ & $2 / 85(2 \%)$ & $1 / 32(3 \%)$ & 0.999 \\
\hline $12 \mathrm{mo}^{\mathrm{d}}(2 / 653.1 ; 2 / 563.6 \mathrm{p}=1.0)$ & $0 / 12(0 \%)$ & $1 / 75(1 \%)$ & $1 / 87(1 \%)$ & $3 / 34(9 \%)$ & 0.120 \\
\hline $2 \mathrm{yrs}^{\mathrm{d}}(3 / 684.4 ; 6 / 6010.0 \mathrm{p}=0.30)$ & $0 / 15(0 \%)$ & $4 / 77(5 \%)$ & $4 / 92(4 \%)$ & $5 / 36(14 \%)$ & 0.130 \\
\hline $5 \mathrm{yrs}^{\mathrm{e}}(11 / 6118.0 ; 11 / 5320.8 \mathrm{p}=0.81)$ & $1 / 16(6 \%)$ & $13 / 71(18 \%)$ & $14 / 87(16 \%)$ & $8 / 27(30 \%)$ & 0.201 \\
\hline
\end{tabular}

Categorical variables: $\mathrm{n} / \mathrm{N}$ yes/total number (\%). Continuous variables: median (interquartile range).

$* \mathrm{p}$ for comparisons of combined anthroposophic and partly anthroposophic versus nonanthroposophic lifestyle group and for girls $v s$ boys regarding sensitization to allergens. Categorical variables: Fisher's exact test; continuous variables: Mann-Whitney-Wilcoxon rank-sum test.

${ }^{\mathrm{a}}$ Classified as sensitized if IgE level was $\geq 0.35 \mathrm{kU}_{\mathrm{A}} / \mathrm{L}$ measured using Phadiatop ${ }^{\mathrm{TM}}$ (Thermo Fisher Scientific) a mix of 11 aeroallergens.

${ }^{\mathrm{b}}$ Classified as sensitized if IgE level was $\geq 0.35 \mathrm{kU}_{\mathrm{A}} / \mathrm{L}$ for at least one of the three food allergens analyzed using ImmunoCAP ${ }^{\mathrm{TM}}$ (Thermo Fisher Scientific).

${ }^{\mathrm{c}}$ Classified as sensitized if IgE level was $\geq 0.35 \mathrm{kU}_{\mathrm{A}} / \mathrm{L}$ for at least one of the six food allergens analyzed using a food mix, fx 5 , followed by separate ImmunoCAP ${ }^{\mathrm{TM}}$ tests (Thermo Fisher Scientific).

${ }^{\mathrm{d}}$ Classified as sensitized if IgE level was $\geq 0.35 \mathrm{kU}_{\mathrm{A}} / \mathrm{L}$ for at least one of the four aeroallergens analyzed using ImmunoCap ${ }^{\mathrm{TM}}$ (Thermo Fisher Scientific).

${ }^{\mathrm{e}}$ Classified as sensitized if IgE level was $\geq 0.35 \mathrm{kU}_{\mathrm{A}} / \mathrm{L}$ for at least one of the 9 aeroallergens analyzed using Phadiatop ${ }^{\mathrm{TM}}$ followed by separate ImmunoCAP ${ }^{\mathrm{TM}}$ tests (Thermo Fisher Scientific).

in Wharton's jelly. Villitis was defined as the presence of mononuclear cell infiltrates in the villous stroma [17].

2.4. Selection of Genes for Acetylation Analyses in Placenta. CD14 (CD14 molecule), FOXP3 (Forkhead box P3), HDAC4 (Histone deacetylase 4), INFG (Interferon gamma), and IL13 (Interleukin 13) were selected based on (1) previous data showing significant differences in DNA methylation between atopic and nonatopic children [18], (2) empirical evidence of differential DNA methylation due to exposure to environmental factors such as farming [13], (3) other studies showing potential allergy-relevant association on the epigenetic level [19, 20], and (4) evidence in various allergyrelated animal models of epigenetic changes that could be transmitted to offspring [21, 22]. SH2B3 (SH2B adaptor protein 3) was selected based on the results of a genome-wide DNA methylation study revealing significant differences in the DNA methylation levels of this gene in purified memory cutaneous lymphocyte-associated antigen (CLA) ${ }^{+} \mathrm{T}$ cells from atopic eczema patients [23].

2.5. Isolation of Chromatin from Snap Frozen Placenta, Chromatin Immunoprecipitation, and Quantitative Polymerase Chain Reaction. A subsection spanning the whole thickness of the placenta was manually obtained in a $-80^{\circ} \mathrm{C}$ freezer from the original sample and then kept for $8 \mathrm{~min}$ in $1 \mathrm{ml} \mathrm{l} \%$ paraformaldehyde (PFA; Sigma-Aldrich, Munich, Germany) 
TABLE 2: Primers used for quantitative assessment of $\mathrm{H} 3$ or $\mathrm{H} 4$ histone acetylation by PCR following chromatin immunoprecipitation (ChIP).

\begin{tabular}{lrc}
\hline Gene & Forward primer & Reverse primer \\
\hline CD14 & ATCAGGGTTCACAGAGGA & GACCCCAAGACCCTACAC \\
\hline FOXP3 & ATCGTGAGGATGGATGCATTAATA & CCACTGGGAAGGTCCCTAGC \\
\hline HDAC4 & CTCAACACAAGCCTCCCAAG & GTGAGGGTGTGGGGTGTAG \\
\hline IL13 & AATCCCACCAGAATGGCACAGGTG & GAACAATGTGCTGCACCTCCTCTGG \\
\hline RPL32 & TGTGGGAGATGCCGTGGG & TCTGACTCCCAGAAGTCTGC \\
\hline SH2B3 & GGAAGTGCTTGCCTTTTTCC & GGATTGCCACGGATTAACAC \\
\hline
\end{tabular}

at room temperature (RT). Next, the sample was centrifuged for $5 \mathrm{~min}$ at 7,870 $\mathrm{g}$ at RT, incubated with $1 \mathrm{ml} \mathrm{0.25 \%}$ trypsinethylenediaminetetraacetic acid (EDTA; Thermo Fisher Scientific, Waltham, MA, USA) for 1 hour at RT and then again centrifuged for $5 \mathrm{~min}$ at 7,870 $\mathrm{g}$ at RT. The supernatant was discarded, and the tissue components were incubated with $0.1 \%$ collagenase (Roche Diagnostics, Mannheim, Germany) for another hour at RT and then centrifuged again for $5 \mathrm{~min}$ at 7,870 $\mathrm{g}$ at RT. To purify cells from tissue remnants and cell debris, the pellet was then resuspended in $1 \mathrm{ml}$ PBS and run through a $0.2 \mu \mathrm{m}$ sieve. Next, the cells were washed twice with $1 \mathrm{ml}$ PBS. Further steps, including chromatin purification, chromatin immunoprecipitation (ChIP), and quantitative assessment of $\mathrm{H} 3$ or $\mathrm{H} 4$ histone acetylation by polymerase chain reaction (PCR), were conducted as established and thoroughly validated before [24]. PCR primers used in the present study are given in (Table 2).

In brief, three-level strategy of PCR data normalization was applied. First, percent enrichment to the input control was calculated for each target locus and a positive control gene encoding ribosomal protein L32 (RPL32), separately for mock (IgG), H3, and H4 antibodies. Then, locus-specific percent enrichment to the input control obtained for IgG was subtracted from the corresponding values for $\mathrm{H} 3$ or $\mathrm{H} 4$ antibodies. Such calculated IgG-corrected percent enrichment was divided for each gene into that of RPL32 resulting in a relative enrichment value, which was used for subsequent statistical analyses $[24,25]$. Intra- and interassay coefficients of variation calculated for percent enrichment should not exceed $10 \%$ [24]. All samples were processed according to the same standardized protocol and analyzed blinded and in a randomized order.

2.6. Statistical Analyses. Due to the limited number of available placenta specimens in the anthroposophic group, this group was merged with the partly anthroposophic group for the statistical analyses. Demographic data were compared between the study subgroups either by Fisher's exact test (binary variables) or Mann-Whitney-Wilcoxon rank sum test (continuous variables). Fisher's exact test was used in the analyses of placenta histopathology in relation to lifestyle and sensitization of the children and their sex. The histone acetylation levels were presented by their median and interquartile range in the different lifestyle groups, and Mann-WhitneyWilcoxon rank sum test was used to compare groups.
Binary variables indicating sensitization to food- or aeroallergens were recorded at 6 months, 1, 2, and 5 years of age. Generalized estimating equations (GEE) were used to compute odds ratios (ORs) associated with histone acetylation levels and the corresponding 95\% CIs. All regressions included dummy variables indicating the time, in order to capture potential nonlinear trends. Additional analyses were performed by further adjusting for the sensitization of the parents. OR reflects the change in the odds of being sensitized associated with a unit increase of the acetylation levels. An OR greater than 1 indicates that the associated predictor may be a risk factor for sensitization; an OR less than 1 suggests that the associated predictor is protective against sensitization; an OR equal to 1 , or not significantly different from it, does not permit establishing an association between histone acetylation levels and the risk of sensitization. The $\mathrm{R}$ package gee, version 4.13-19 (https://cran.r-project.org/web/packages/gee/), was used for the analysis. The analyses were repeated stratifying by gender and, separately, by lifestyle. A p value $<0.010$ was considered significant. Model-based receiver operating characteristic (ROC) curves were drawn to test for the ability to predict sensitization to allergens and the area under the curve (AUC) was calculated as a measure of performance, using the GEE logit model described above.

\section{Results}

3.1. Study Population. Comparison of the lifestyle groups showed significant differences for the anthroposophic (anthroposophic + partly anthroposophic) lifestyle characteristics compared with the nonanthroposophic group regarding a lower prevalence of sensitization, particularly to food allergens, in the children (Table 1).

3.2. Placenta Histopathology. Placental histopathology showed neither significant differences between the two-lifestyle groups (Table 3(a)) nor any associations with sensitization to allergens in the children between 6 months up to 5 years of age (Table 3(b)), irrespective of the child's sex (Supplementary Tables $1 \mathrm{~A}$ and $1 \mathrm{~B})$.

3.3. Associations between Demographic Data and Placental Histone Acetylation. The age of the mother, parity or parental 


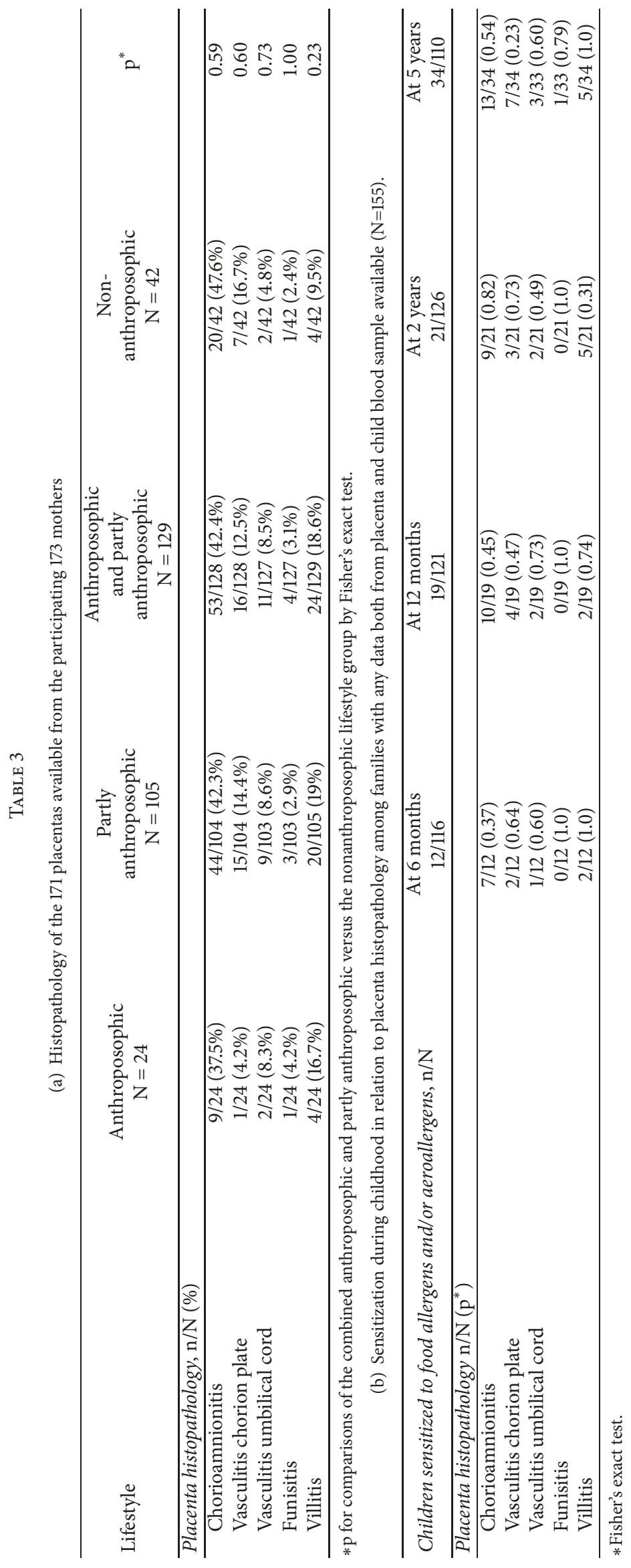



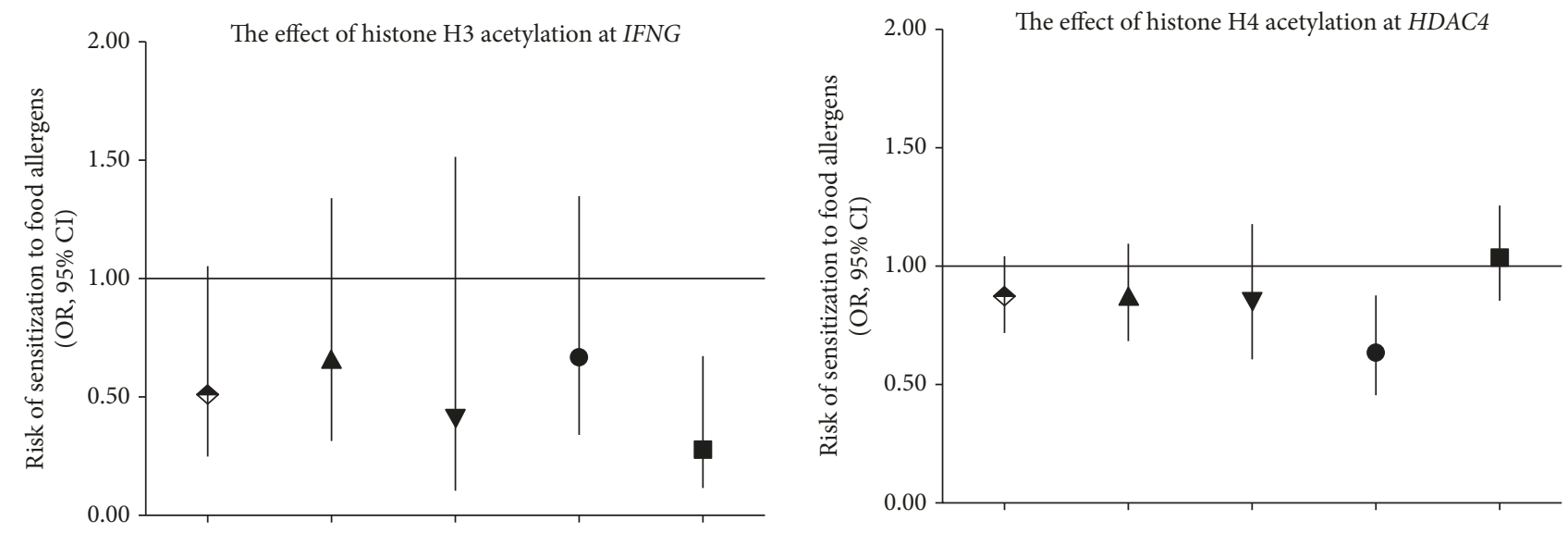

\begin{tabular}{|l|}
\hline $\mathrm{p}$ \\
\hline Group
\end{tabular}$\quad$\begin{tabular}{|c|c|c|c|c|}
\hline 0.066 & 0.250 & 0.182 & 0.262 & 0.004 \\
\hline All & $\begin{array}{c}\text { Anth }+ \\
\text { Part-anth }\end{array}$ & $\begin{array}{c}\text { Non- } \\
\text { anth }\end{array}$ & Girls & Boys \\
\hline
\end{tabular}

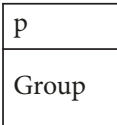

\begin{tabular}{|c|c|c|c|c|}
\hline 0.128 & 0.230 & 0.313 & 0.005 & 0.774 \\
\hline All & $\begin{array}{c}\text { Anth + } \\
\text { Part-anth }\end{array}$ & $\begin{array}{c}\text { Non- } \\
\text { anth }\end{array}$ & Girls & Boys \\
\hline
\end{tabular}

(b)

(a)

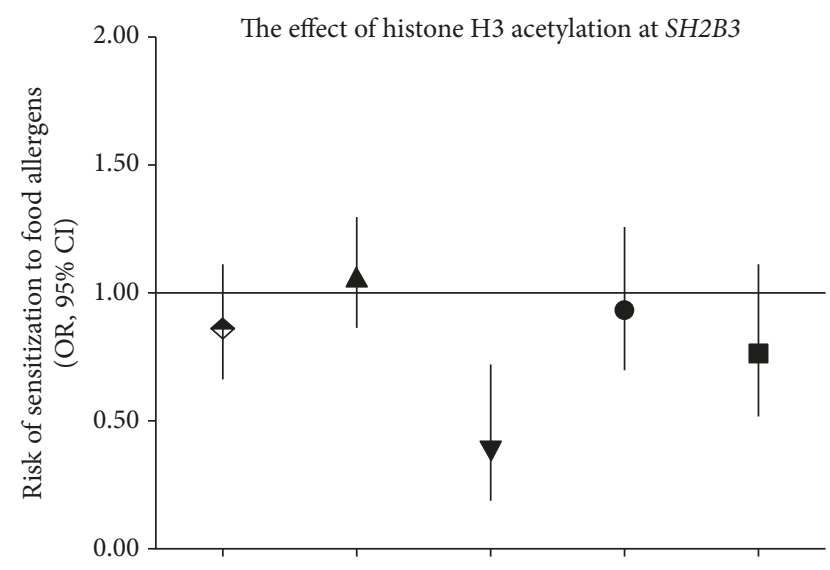

\begin{tabular}{|c|c|c|c|c|c|}
\hline p & 0.248 & 0.571 & 0.003 & 0.645 & 0.151 \\
\hline Group & All & $\begin{array}{c}\text { Anth + } \\
\text { Part-anth }\end{array}$ & $\begin{array}{l}\text { Non- } \\
\text { anth }\end{array}$ & Girls & Boys \\
\hline
\end{tabular}

(c)

Figure 1: The association between placental tissue histone acetylation levels at promoter regions of (a) IFNG (H3), (b) HDAC4 (H4), and (c) SH2B3 (H3) genes and the risk of sensitization to food allergens in children. For the methodology of statistical calculations, please, see Methods. Anth + Part-anth denotes a combined anthroposophic and partly anthroposophic lifestyle group and Non-anth the nonanthroposophic lifestyle. OR denotes odds ratio; 95\% CI, confidence interval.

sensitization to aeroallergens showed no significant associations with histone acetylation levels in the placentas, nor did the child's sex, birth weight, or the gestational age at delivery (data not shown). In addition, there was no significant effect of the lifestyle on the placental histone acetylation levels and also none when prestratified for the child's sex (Supplementary Table 2).

3.4. Placental Histone Acetylation and Reduced Risk of Allergic Sensitization in the Child. Placental histone acetylation levels at the promoter regions of 3 genes, IFNG, HDAC4, and
SH2B3, turned out to be predictive for the development of allergic sensitization in the children followed longitudinally from 6 months up to 5 years of age. Decreased risk of sensitization to food allergens was associated with higher $\mathrm{H} 3$ acetylation levels in placentas at the IFNG loci in male offspring and to a higher $\mathrm{H} 4$ acetylation at the HDAC4 promoter in female offspring (Figures 1(a) and 1(b)). In addition, higher $\mathrm{H} 3$ acetylation at the $\mathrm{SH} 2 \mathrm{~B} 3$ locus was associated with a decreased risk of sensitization to food allergens in children born in nonanthroposophic families (Figure 1(c)). Regarding sensitization to aeroallergens, a higher $\mathrm{H} 4$ acetylation level at the HDAC4 promoter decreased the risk of sensitization in 


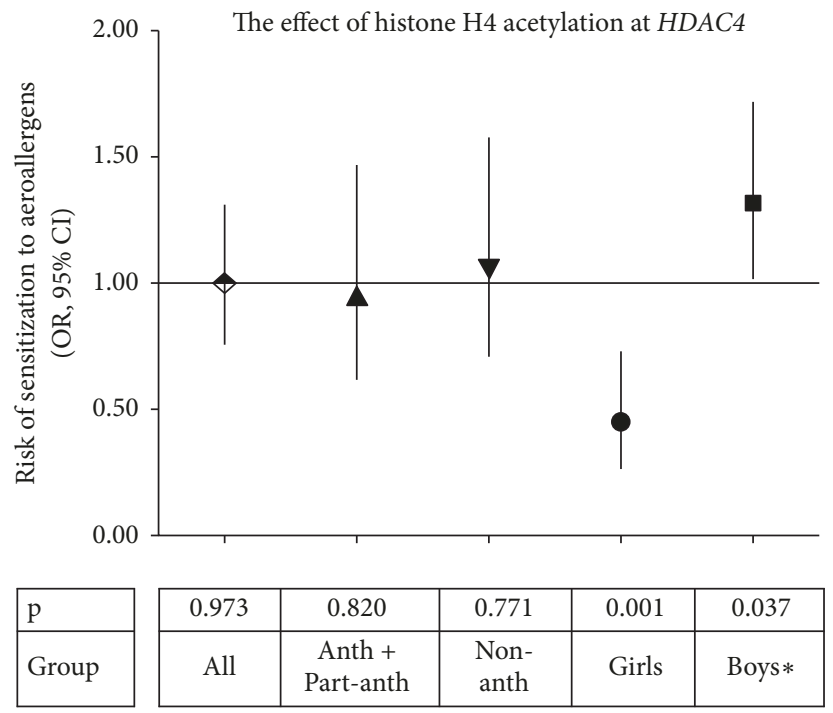

FIGURE 2: The association between placental tissue histone $\mathrm{H} 4$ acetylation levels at promoter region of the HDAC4 gene and the risk of sensitization to aeroallergens in children. For the methodology of statistical calculations, please, see Methods. For abbreviations, see Figure 1. * Calculated with logistic regression since the number of observations in the group of boys precluded the estimations of OR and CI with the GEE model.

female offspring (Figure 2). All these associations remained significant after adjustment to either maternal or paternal allergic sensitization. We did not observe any significant associations between histone acetylation levels at the CD14, IL13, and FOXP3 promoters and the development of sensitization to allergens (data not shown).

Next, we computed model-based ROC curves to see how well the used regression models can predict sensitization to allergens and computed AUC as a measure of performance. On the left panels in Figure 3, we report the following ROC curves: $\mathrm{H} 3$ acetylation at the IFNG promoter versus sensitization to food allergens in boys (Figure 3(a)), H4 acetylation at the HDAC4 promoter in girls versus sensitization to food allergens in girls (Figure 3(b)), H3 acetylation at the $\mathrm{SH} 2 \mathrm{~B} 3$ locus versus sensitization to food allergens in nonanthroposophic children (Figure 3(c)), and $\mathrm{H} 4$ acetylation at the HDAC4 promoter versus sensitization to aeroallergens in girls (Figure 3(d)). The ROC curves and the AUC suggest that the predictive power of the models is rather limited with the highest AUC level of 0.777 (Figure 3(d)). In the right panels in Figure 3, the ROC curves obtained with the GEE logistic model are further adjusted for the other histone acetylation variables showing any significant associations with sensitization to allergens (see Figures 1 or 2, respectively). Results show that multiple adjustment does not significantly improve the predictive power.

The dataset for this study including demographic data, sensitization to allergens, and histone acetylation levels can be found in the Supplementary data table.

\section{Discussion}

This is the first study linking the development of sensitization to allergens early in life with defined changes in the histone acetylation of important immunoregulatory genes in the placenta. We have discovered significant associations between histone acetylation levels in 3 of the 6 allergy candidate genes examined in placentas with the development of sensitization to allergens.

Higher placental histone acetylation levels were associated with decreased risk of allergic sensitization to food allergens in children. This involved $\mathrm{H} 3$ acetylation in the IFNG gene, $\mathrm{H} 4$ acetylation in $\mathrm{HDAC4}$, and $\mathrm{H} 3$ acetylation in SH2B3. Furthermore, $\mathrm{H} 4$ acetylation in HDAC4 was also associated with a decreased risk of allergic sensitization to aeroallergens. These findings revealed $\mathrm{HDAC} 4$ and $\mathrm{SH} 2 \mathrm{~B} 3$ as two new candidates implicated in the susceptibility to allergic sensitization. The protein encoded by HDAC4 possesses histone deacetylase activity and represses transcription when tethered to a promoter $[26,27]$. This protein does not bind to DNA directly, but through transcription factors MEF2C and MEF2D and it seems to interact in a multiprotein complex with HDAC3 and RB binding protein 4 (RBBP4), a molecule which may target histone deacetylases to their histone substrates [26]. HDAC4 is of great interest in B cell biology since forced expression of HDAC4 impairs the inflammatory effects of miRNA-155 in this cell [28] and because $B$ cell functions seems to be particularly sensitive to HDAC inhibitors [29]. HDAC4 also provides deacetylase activity for nonhistone proteins in the cytoplasm including signal transducer and activator of transcription 1 (STAT1), a protein that promotes interferon signaling pathways [30]. We speculate that increased $\mathrm{H} 4$ acetylation of $H D A C 4$ detected in this study may lead to changes in HDAC4 expression in placenta and by genome-wide deacetylase effects alter the expression of other immune genes and/or transcription factors involved in Th1-skewing which in turns facilitates early immune polarization and could confer protection from 

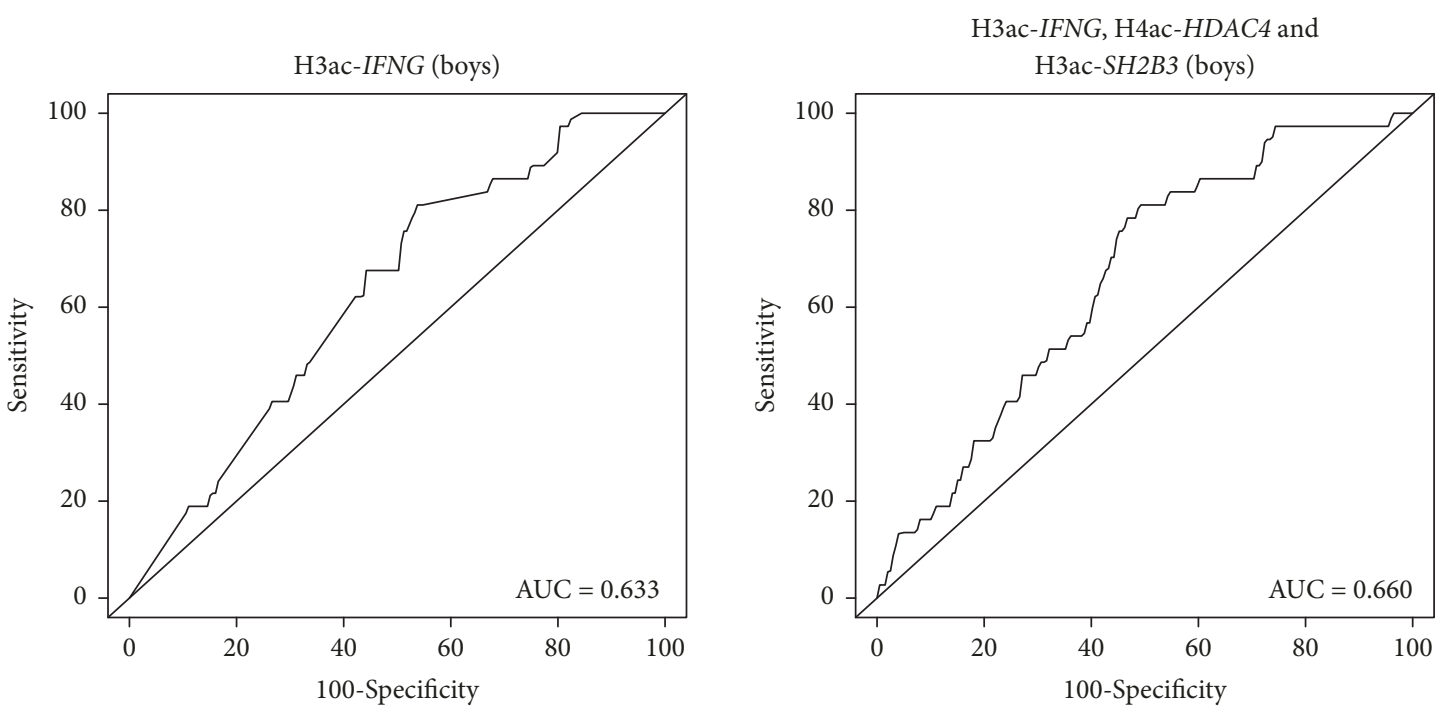

(a) Sensitization to food allergens
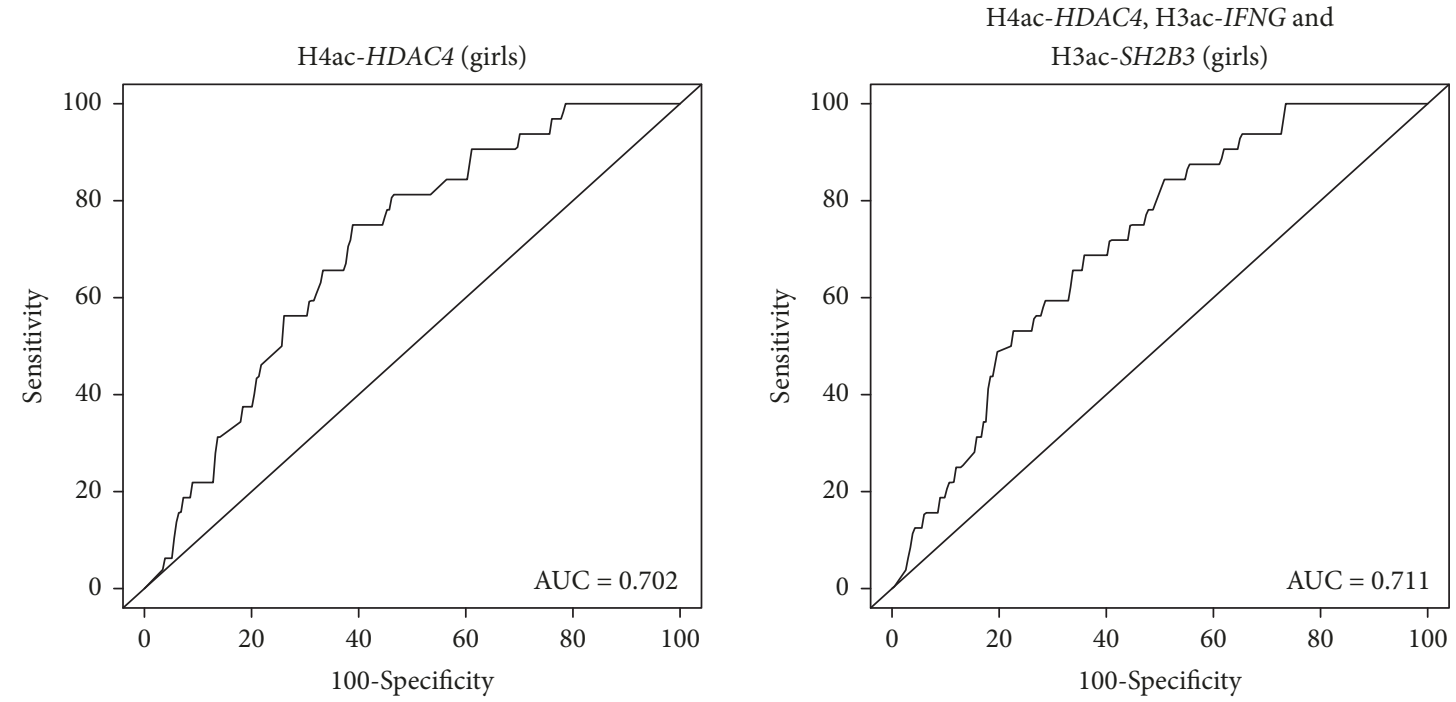

(b) Sensitization to food allergens
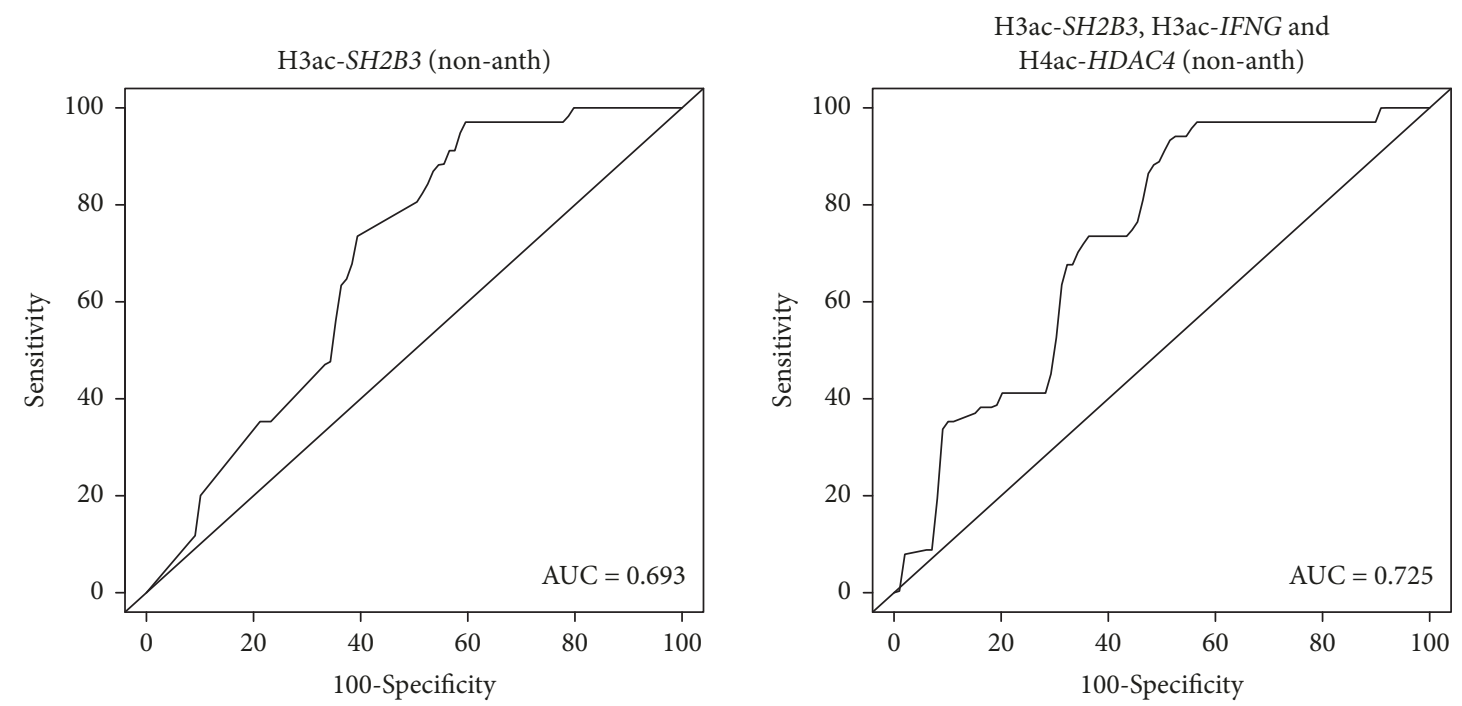

(c) Sensitization to food allergens

Figure 3: Continued. 

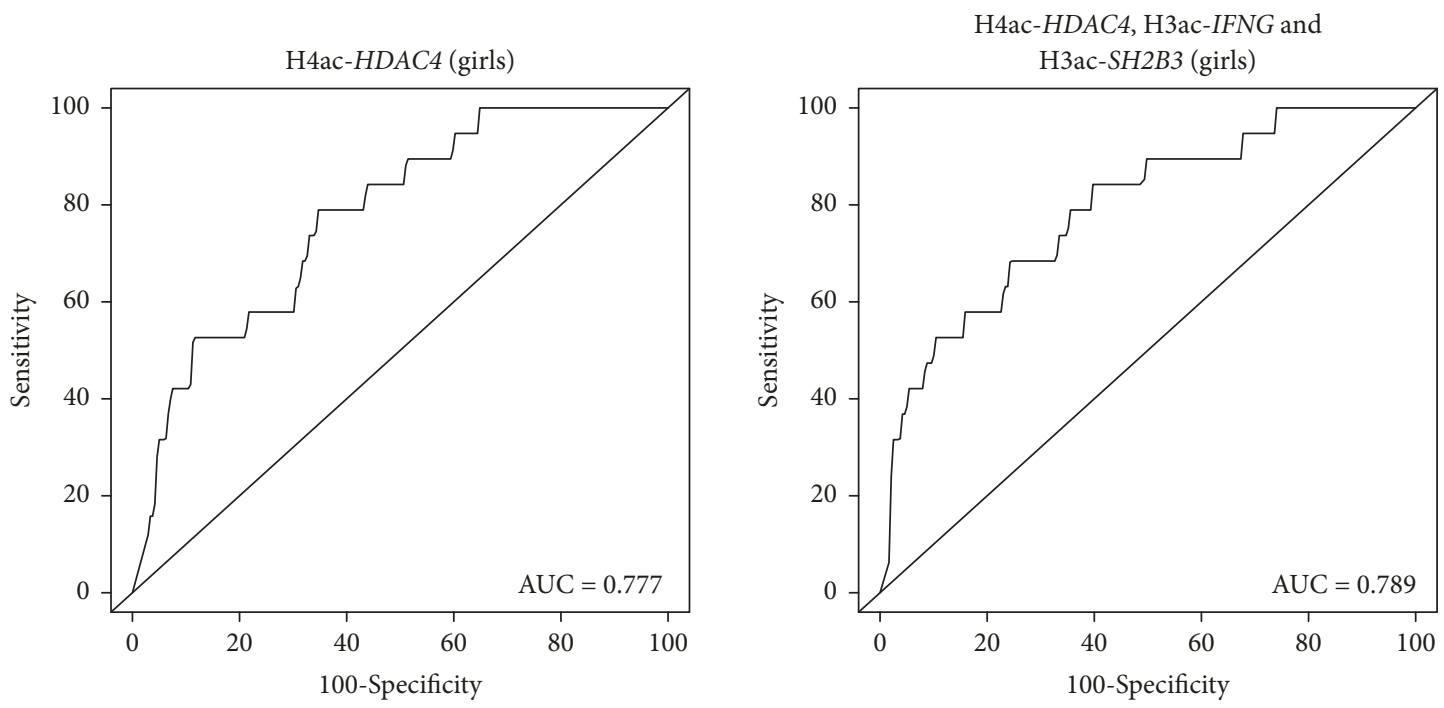

(d) Sensitization to aeroallergens

FIGURE 3: Receiver operating characteristic (ROC) curves and area under the curve (AUC) obtained from different logistic regression models implemented through GEE to predict the development of sensitization to allergens. Left panel: (a) H3 acetylation (H3ac) at the IFNG promoter versus sensitization to food allergens in boys, (b) $\mathrm{H} 4$ acetylation ( $\mathrm{H} 4 \mathrm{ac}$ ) at the HDAC4 promoter in girls versus sensitization to food allergens in girls, (c) H3 acetylation (H3ac) at the SH2B3 locus versus sensitization to food allergens in nonanthroposophic children (non-anth), and (d) $\mathrm{H} 4$ acetylation (H4ac) at the HDAC4 promoter versus sensitization to aeroallergens in girls. Right panel: (a-d), analogous prediction obtained when the GEE logistic model is further adjusted for the other histone acetylation variables showing significant associations with sensitization to allergens, as indicated (see also Figures 1 and 2).

IgE sensitization in the offspring. HDAC4 is an age-modified locus [26] and has shown to be susceptible to epigenetic modification by environmental exposures including supplementation with n-3 PUFAs [31].

$\mathrm{SH} 2 \mathrm{~B} 3$ encodes a member of the $\mathrm{SH} 2 \mathrm{~B}$ adaptor family of proteins that acts as a key negative regulator of cytokine signaling. It plays a critical role in lymphohematopoiesis, inflammation [32], and IL7R signaling in B cell progenitors [33]. It also regulates granulocyte-macrophage colony-stimulating factor (GM-CSF) and interleukin 15 (IL-15) signals in mature dendritic cells (DCs) and affects their ability to prime naive $\mathrm{CD}^{+} \mathrm{T}$ cells towards IFNG production [34]. Our results support significant association of acetylation differences in the IFNG gene in placenta and the decreased risk of allergic sensitization, particularly to food allergens (Figure 1(a)). Immune-homeostasis after birth depends on a rapid development of strong $\mathrm{T}$ helper type 1 (Th1) responses. These are needed to combat extracellular and intracellular pathogens and help to keep the development of pathogenic Th2 immune responses down. It has previously been shown [33, 35, 36] that children protected from the development of (respiratory) allergy have already, at birth, high levels/production of Th1 related cytokines, including IFNG. We are now able to extend these observations and show for the first time that already during pregnancy such a response seems to develop at least at the late stage of gestation in the placental tissue.

Several of our observations were gender-specific; for instance, $\mathrm{H} 4$ acetylation differences were only significant for girls, in agreement with accumulating evidence on genderspecific effects of environmental exposures in placenta. Sexspecific epigenetic effects have been also reported in the context of allergies by others, but a functional and mechanistic explanation for these findings is still missing $[37,38]$.

A limitation in this study is that only 25 anthroposophic families could be included due to the amount of available snap frozen placenta specimens in this life style group (Table 1). We therefore fused the anthroposophic group with the partly anthroposophic group for the statistical analyses that is why separate life style analyses was hampered. Another limitation is the use of placenta tissue, where the cell heterogeneity in the samples does not allow any interpretation to which particular cells the epigenetic profiles of $\mathrm{H} 4$ and $\mathrm{H} 3$ acetylation could be ascribed. We performed, however, careful placenta histopathology examinations to address any bias due to inflammation and could exclude any significant differences in the presence and distribution of leukocytes between the lifestyle groups or any associations to sensitization to allergens in the children (see Tables 3(a) and 3(b)). Furthermore, since we analyzed global $\mathrm{H} 3$ and $\mathrm{H} 4$ acetylation levels in each of the genes it is not possible to discriminate specific marks, which underlie the associations detected in this study. The combinations of genes and histone acetylation marks analyzed in this study demonstrated rather moderate predictive value for the development of sensitization to allergens. Deeper and fine-tuned mapping of histone marks as well as whole genome sequencing is needed to delineate the exact predictive effect of different genes. 


\section{Conclusions}

Our results indicate that histone acetylation levels in allergyrelated immune regulatory genes in placenta might have a potential to predict the development of sensitization to allergens in the child within the first 5 years after birth. The epigenetic profiles shown here for the acetylation in the $\mathrm{H} 3$ and $\mathrm{H} 4$ histones may open new preventive avenues and lay the foundation for further prospective studies.

\section{Data Availability}

Demographic and histone acetylation data used to support the findings of this study are available as Supplementary data table and the histopathologic data are available from the corresponding author upon request.

\section{Conflicts of Interest}

J. Alm has served as a consultant on clinical trials for ALK-Abello. A. Scheynius is a member in the Joint Steering Committee for the Human Translational Microbiome Program at SciLifeLab/Karolinska Institutet together with Ferring Pharmaceuticals, Switzerland. The rest of the authors declare that they have no relevant conflicts of interest.

\section{Acknowledgments}

We acknowledge the families participating in the ALADDIN study for their trust and contribution and the ALADDIN team for their involvement in this work, especially nurse and coordinator Margareta Eriksson, medical doctor Fredrik Stenius, and biomedical analysts Monica Nordlund and Carina Wallén. We thank Matteo Bottai, Karolinska Institutet, for statistical advice. This study was supported by the Swedish Research Council (2012-3011), the German Center for Lung Research (DZL; 82DZL00502/A2), the Swedish Research Council for Working Life and Social Research (2006-1630), the regional agreement on medical training and clinical research (ALF) between Stockholm County Council and the Karolinska Institutet and the Karolinska University Hospital, the King Gustaf V:s 80-årsfond (FAA-2015-0151), the Swedish Society of Medicine, the Center for Allergy Research Karolinska Institutet, the Mjölkdroppen Society, the Swedish Asthma and Allergy Research Association, the Cancer and Allergy Fund, the Ekhaga Foundation, the Frimurare Barnhuset Foundation in Stockholm, the Hesselman Foundation, the Samariten Foundation, the Vardal Foundation, the Universities Giessen and Marburg Lung Center (UGMLC), the Von Behring-Röntgen-Foundation (Von Behring-RöntgenStiftung; 62-LV04), and the German Academic Exchange Service (DAAD; B Alashkar Alhamwe, Personal Reference no. 91559386).

\section{Supplementary Materials}

Supplementary Table 1 A. Histopathology according to sex of the child. Supplementary Table 1 B. Sensitization during childhood according to sex of the child in relation to placenta histopathology. Supplementary Table 2. Histone acetylation levels in 173 placentas in the different lifestyle groups, and in girls and boys within each group. Supplementary data table. Demographic and histone acetylation data (database). (Supplementary Materials)

\section{References}

[1] C. Brooks, N. Pearce, and J. Douwes, "The hygiene hypothesis in allergy and asthma: an update," Current Opinion in Allergy and Clinical Immunology, vol. 13, no. 1, pp. 70-77, 2013.

[2] S. H. Christensen, S. Timm, C. Janson et al., "A clear urbanrural gradient of allergic rhinitis in a population-based study in Northern Europe," European Clinical Respiratory Journal, vol. 3, no. 1, p. 33463, 2016.

[3] A. DeVries and D. Vercelli, "Epigenetics in allergic diseases," Current Opinion in Pediatrics, vol. 27, no. 6, pp. 719-723, 2015.

[4] M. Kabesch, "Early origins of asthma (and allergy)," Molecular and Cellular Pediatrics, vol. 3, no. 1, p. 31, 2016.

[5] D. P. Potaczek, H. Harb, S. Michel, B. A. Alhamwe, H. Renz, and J. Tost, "Epigenetics and allergy: from basic mechanisms to clinical applications," Epigenomics, vol. 9, no. 4, pp. 539-571, 2017.

[6] H. Harb, B. Alashkar Alhamwe, H. Garn, H. Renz, and D. P. Potaczek, "Recent developments in epigenetics of pediatric asthma," Current Opinion in Pediatrics, vol. 28, no. 6, pp. 754763, 2016.

[7] P. O. Brook, M. M. Perry, I. M. Adcock, and A. L. Durham, "Epigenome-modifying tools in asthma," Epigenomics, vol. 7, no. 6, pp. 1017-1032, 2015.

[8] M. Kabesch, S. Michel, and J. Tost, "Epigenetic mechanisms and the relationship to childhood asthma," European Respiratory Journal, vol. 36, no. 4, pp. 950-961, 2010.

[9] H. Renz, P. Brandtzaeg, and M. Hornef, "The impact of perinatal immune development on mucosal homeostasis and chronic inflammation," Nature Reviews Immunology, vol. 12, no. 1, pp. 9-23, 2012.

[10] B. Alaskhar Alhamwe, R. Khalaila, J. Wolf et al., "Histone modifications and their role in epigenetics of atopy and allergic diseases," Allergy, Asthma \& Clinical Immunology, vol. 14, p. 39, 2018.

[11] S. Ashley, T. Dang, J. Koplin, D. Martino, and S. Prescott, "Food for thought: progress in understanding the causes and mechanisms of food allergy," Current Opinion in Allergy and Clinical Immunology, vol. 15, no. 3, pp. 237-242, 2015.

[12] I. V. Yang, C. A. Lozupone, and D. A. Schwartz, "The environment, epigenome, and asthma," Journal of Allergy and Clinical Immunology, vol. 140, no. 1, pp. 14-23, 2017.

[13] G. G. Slaats, L. E. Reinius, J. Alm, J. Kere, A. Scheynius, and M. Joerink, "DNA methylation levels within the CD14 promoter region are lower in placentas of mothers living on a farm," Allergy, vol. 67, no. 7, pp. 895-903, 2012.

[14] P. E. Fields, S. T. Kim, and R. A. Flavell, "Cutting edge: changes in histone acetylation at the IL- 4 and IFN-gamma loci accompany Th1/Th2 differentiation," Journal of Immunology, vol. 169, no. 2, pp. 647-650, 2002.

[15] F. Stenius, J. Swartz, G. Lilja et al., "Lifestyle factors and sensitization in children - the ALADDIN birth cohort," Allergy, vol. 66, no. 10, pp. 1330-1338, 2011. 
[16] J. S. Alm, J. Swartz, G. Lilja, A. Scheynius, and G. Pershagen, "Atopy in children of families with an anthroposophic lifestyle," The Lancet, vol. 353, no. 9163, pp. 1485-1488, 1999.

[17] I. Hulthén Varli, M. Kublickas, N. Papadogiannakis, and K. Petersson, "Chorioamnionitis without foetal inflammatory response is associated with stillbirth in early preterm pregnancies," The Journal of Maternal-Fetal \& Neonatal Medicine, vol. 26, no. 10, pp. 953-959, 2013.

[18] J. Nicodemus-Johnson, K. A. Naughton, J. Sudi et al., "Genomewide methylation study identifies an IL-13-induced epigenetic signature in asthmatic airways," American Journal of Respiratory and Critical Care Medicine, vol. 193, no. 4, pp. 376-385, 2016.

[19] S. Khoo, M. Mäkelä, D. Chandler et al., "No simple answers for the Finnish and Russian Karelia allergy contrast: Methylation of CD14 gene," Pediatric Allergy and Immunology, vol. 27, no. 7, pp. 721-727, 2016.

[20] I. U. Ogbuanu, W. J. Karmaus, H. Zhang et al., "Birth order modifies the effect of IL13 gene polymorphisms on serum IgE at age 10 and skin prick test at ages 4,10 and 18: a prospective birth cohort study," Allergy, Asthma \& Clinical Immunology, vol. 6, no. 1, p. 6, 2010.

[21] S. Brand, D. A. Kesper, R. Teich et al., "DNA methylation of TH1/TH2 cytokine genes affects sensitization and progress of experimental asthma," The Journal of Allergy and Clinical Immunology, vol. 129, no. 6, pp. 1602-1610, 2012.

[22] S. Brand, R. Teich, T. Dicke et al., "Epigenetic regulation in murine offspring as a novel mechanism for transmaternal asthma protection induced by microbes," The Journal of Allergy and Clinical Immunology, vol. 128, no. 3, pp. 618-625, 2011.

[23] S. Katayama, S. Bruhn, A. Scheynius, L. Lunderberg, J. Kere, and A. Andersson, "Epigenetic modifications in skin-homing CD4+CLA+ T-cells of atopic dermatitis patients relate to mRNA expression changes in high mobility group proteins," in Proceedings of the 2nd Inflammatory Skin Disease Summit, Experimental Dermatology, pp. 36-37, New York, NY, USA, 2016.

[24] H. Harb, M. Amarasekera, S. Ashley et al., "Epigenetic regulation in early childhood: a miniaturized and validated method to assess histone acetylation," International Archives of Allergy and Immunology, vol. 168, no. 3, pp. 173-181, 2016.

[25] M. Haring, S. Offermann, T. Danker, I. Horst, C. Peterhansel, and M. Stam, "Chromatin immunoprecipitation: optimization, quantitative analysis and data normalization," Plant Methods, vol. 3, no. 1, p. 11.

[26] Z. Wang, G. Qin, and T. C. Zhao, "HDAC4: mechanism of regulation and biological functions," Epigenomics, vol. 6, no. 1, pp. 139-150, 2014.

[27] P. Wawrzyniak, M. Wawrzyniak, K. Wanke et al., "Regulation of bronchial epithelial barrier integrity by type 2 cytokines and histone deacetylases in asthmatic patients," The Journal of Allergy and Clinical Immunology, vol. 139, no. 1, pp. 93-103, 2017.

[28] S. K. Sandhu, S. Volinia, S. Costinean et al., "miR-155 targets histone deacetylase 4 (HDAC4) and impairs transcriptional activity of B-cell lymphoma 6 (BCL6) in the Emu-miR155 transgenic mouse model," in Proceedings of the National Academy of Sciences of the United States of America, vol. 109, pp. 20047-20052, 2012.

[29] M. Waibel, A. J. Christiansen, M. L. Hibbs et al., "Manipulation of B-cell responses with histone deacetylase inhibitors," Nature Communications, vol. 6, 2015.
[30] E. A. Stronach, A. Alfraidi, N. Rama et al., "HDAC4-regulated STAT1 activation mediates platinum resistance in ovarian cancer," Cancer Research, vol. 71, no. 13, pp. 4412-4422, 2011.

[31] B. L. Tremblay, F. Guénard, I. Rudkowska, S. Lemieux, P. Couture, and M. Vohl, "Epigenetic changes in blood leukocytes following an omega-3 fatty acid supplementation," Clinical Epigenetics, vol. 9, no. 43, 2017.

[32] J. Devallière and B. Charreau, "The adaptor Lnk (SH2B3): An emerging regulator in vascular cells and a link between immune and inflammatory signaling," Biochemical Pharmacology, vol. 82, no. 10, pp. 1391-1402, 2011.

[33] Y. Cheng, K. Chikwava, C. Wu et al., "LNK/SH2B3 regulates IL7 receptor signaling in normal and malignant B-progenitors," The Journal of Clinical Investigation, vol. 126, no. 4, pp. 12671281, 2016.

[34] R. N. Beaumont, N. M. Warrington, A. Cavadino et al., "Genome-wide association study of offspring birth weight in 86577 women identifies five novel loci and highlights maternal genetic effects that are independent of fetal genetics," Human Molecular Genetics, vol. 27, no. 4, pp. 742-756, 2018.

[35] D. D. Chaplin, “Overview of the immune response," Journal of Allergy and Clinical Immunology, vol. 125, 2, pp. S3-S23, 2010.

[36] D. F. Gudbjartsson, U. S. Bjornsdottir, E. Halapi et al., "Sequence variants affecting eosinophil numbers associate with asthma and myocardial infarction," Nature Genetics, vol. 41, no. 3, pp. 342-347, 2009.

[37] C. A. Maloney, S. M. Hay, L. E. Young, K. D. Sinclair, and W. D. Rees, "A methyl-deficient diet fed to rat dams during the periconception period programs glucose homeostasis in adult male but not female offspring," Journal of Nutrition, vol. 141, pp. 95100, 2011.

[38] R. Penailillo, A. Guajardo, M. Llanos, S. Hirsch, and AM. Ronco, "Folic acid supplementation during pregnancy induces sexspecific changes in methylation and expression of placental 1lbeta-hydroxysteroid dehydrogenase 2 in rats," PLoS One, vol. 10, no. 3, Article ID e0121098, 2015. 


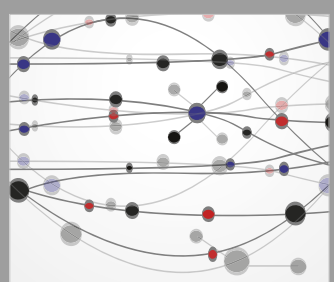

The Scientific World Journal
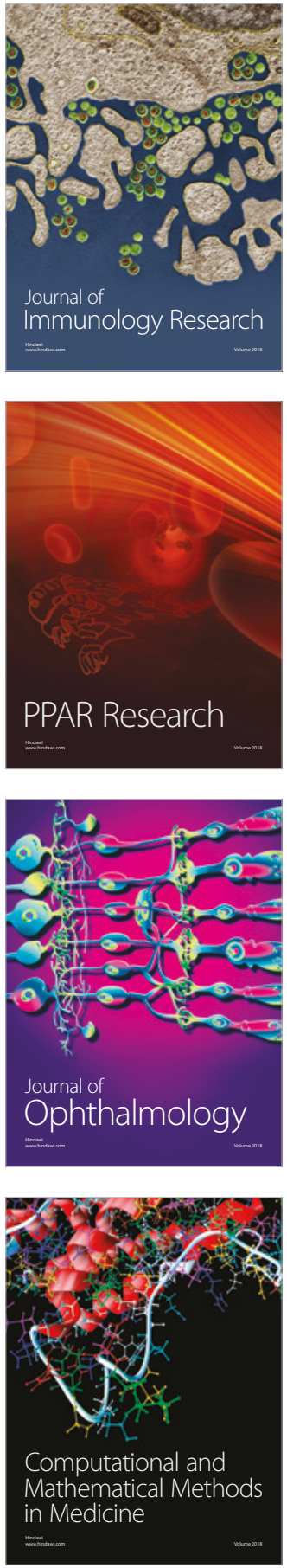

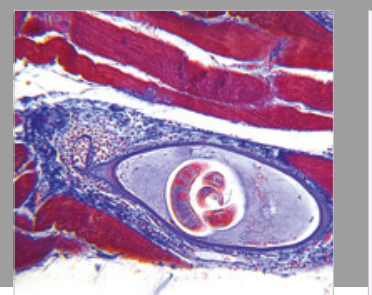

Gastroenterology Research and Practice

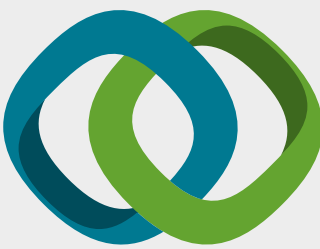

\section{Hindawi}

Submit your manuscripts at

www.hindawi.com
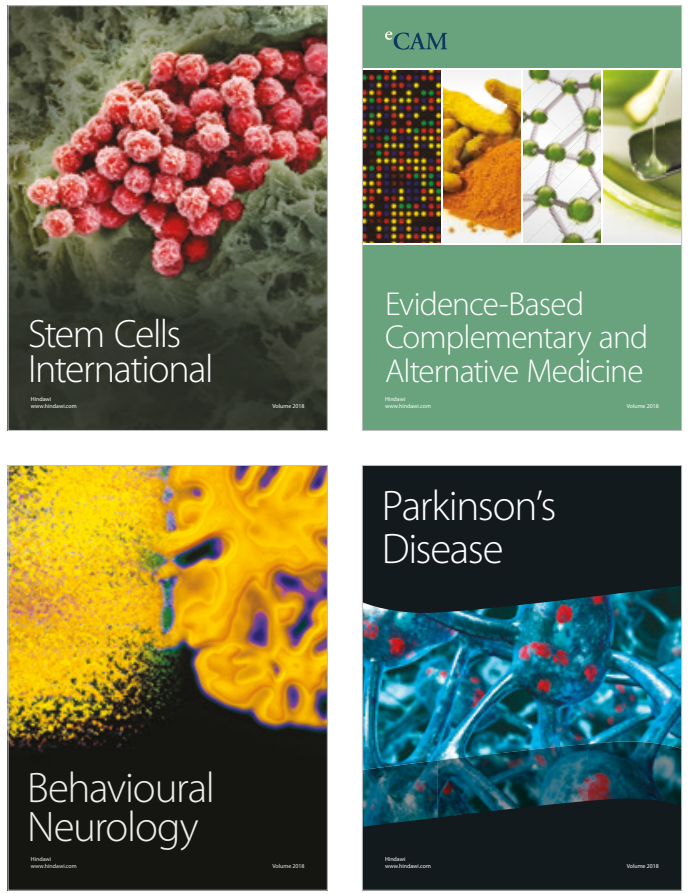

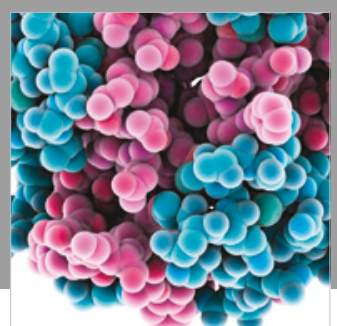

ournal of

Diabetes Research

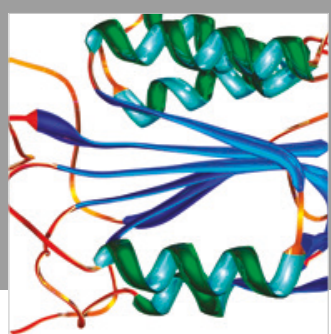

Disease Markers
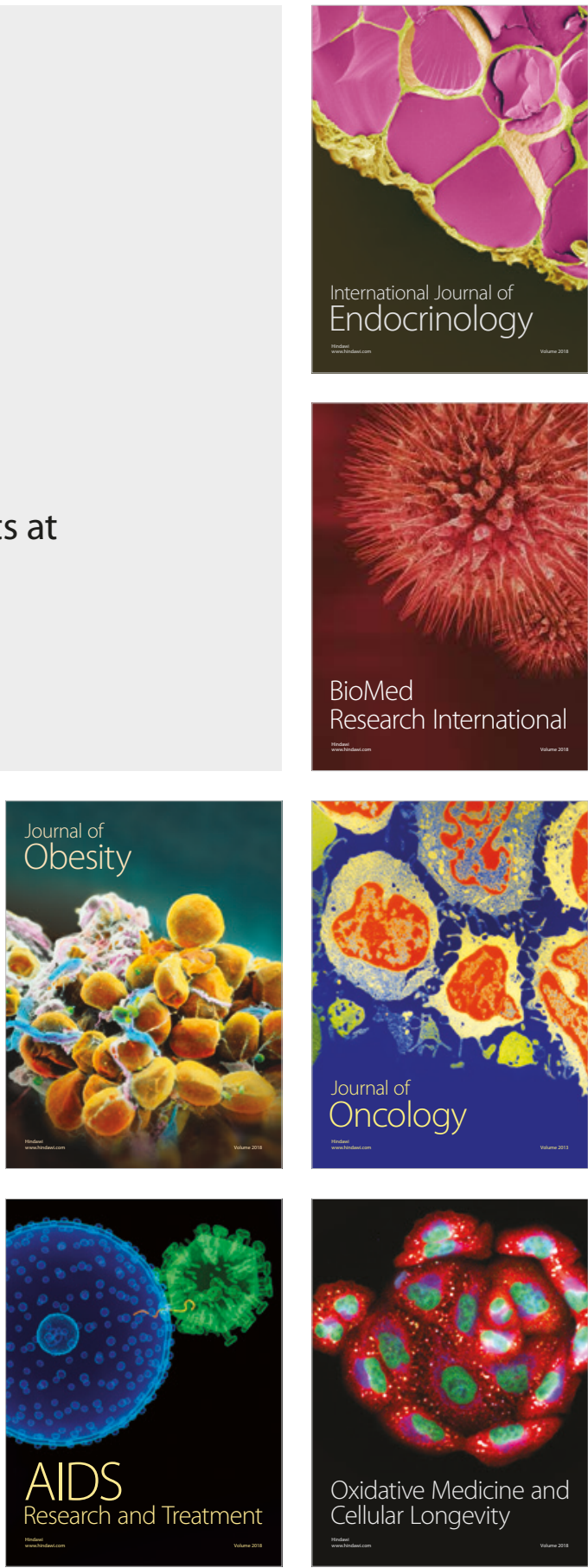